\title{
Prior SARS-CoV-2 infection increases reactogenicity after SARS-COV-2 $m R N A$ vaccine and could delay the administration of the vaccine based on timing of infection
}

\author{
Roberto Stellini $^{1}$ (1) $\cdot$ Renzo Gianello $^{2} \cdot$ Angelo Meloni $^{2,4} \cdot$ Elia Croce $^{4} \cdot$ Paola Materzanini $^{3} \cdot$ Walter Gomarasca $^{4}$
}

Received: 26 July 2021 / Accepted: 6 November 2021 / Published online: 2 December 2021

(c) The Author(s), under exclusive licence to Springer-Verlag GmbH Germany 2021

To the Editor,

Severe Acute Respiratory Syndrome coronavirus 2 (SARS$\mathrm{CoV}-2$ ) infection produces a persistent and protective immune response for at least 6 months in most cases [1] and the messenger RNA (mRNA) vaccines produce a protective immune response via antibodies against the viral receptor-binding domain (RBD) spike (S)-protein. This immune response may differ from individuals with prior or without SARS-CoV-2 infection. These antibodies are related to protection against SARS-CoV-2 infection and especially against moderate/severe SARS-CoV-2 syndrome; in fact, RBD S-Protein IgG is related to the level of neutralizing antibodies and which are protective. This retrospective observational study was designed to verify whether the full vaccine schedule of BNT162b2/Pfizer was really necessary in previously infected individuals and whether the timing of prior infection could influence the vaccine response.

Before the first dose of BNT162b2/Pfizer vaccine, the antibodies against SARS-CoV-2 nucleocapsid (SARS-CoV2N-Protein IgG) (Abbott Alinity Abbott Diagnostics) were measured in Health Care Workers (HCWs) at the Poliambulanza Foundation Hospital Brescia, a 600-bed tertiary care hospital in Northern Italy. The positive threshold was 1.4 signal to cutoff ratio.

Roberto Stellini

roberto.stellini@poliambulanza.it

1 Infectious Diseases Service, Health Care Department, Poliambulanza Foundation Hospital, Brescia, Italy

2 Department of Clinical Laboratory, Poliambulanza Foundation Hospital, Brescia, Italy

3 Department of Occupational Medicine, Poliambulanza Foundation Hospital, Brescia, Italy

4 Health Care Department, Poliambulanza Foundation Hospital, Brescia, Italy
In this study, 45 days after the second dose of the vaccine, the levels of antibodies $\operatorname{IgG}$ against the spike protein receptor-binding domain were quantified using anti SARSCoV-2 IgG II Quant assay (RBD S-Protein IgG) (Abbott Diagnostics). The positive threshold was 50 Arbitrary Units per $\mathrm{mL}(\mathrm{AU} / \mathrm{mL})$ and the upper limit was $40,000 \mathrm{AU} / \mathrm{mL}$, extendable to $80,000 \mathrm{AU} / \mathrm{mL}$ after automatic dilution.

Reverse transcriptase-polymerase chain reaction (RTPCR) was carried out on nasopharyngeal swabs.

Comparisons of quantitative variables, between the various groups, were performed using the Student's $T$ test and Mann-Whitney $U$ test. Two-tailed test was performed, and a $p$ value $<0.001$ was considered statistically significant.

In this retrospective observational cohort study, $1226 / 1510(81.2 \%)$ HCWs had no prior SARS-CoV-2 infection while 284/1510 (18.8\%) had a previously documented SARS-CoV-2 infection ascertained by SARS-CoV$2 \mathrm{~N}-$ Protein IgG positivity. Of these 284 cases, 101 (35.6\%) presented symptomatic infection and they had a positive RTPCR result on nasopharyngeal swabs as well as N-Protein IgG positivity; in contrast, 183/284 (64.4\%) had an asymptomatic previous infection documented only by N-Protein IgG positivity. All the symptomatic and asymptomatic infections occurred prior to vaccination during the first wave (from 21 February to 11 June 2020) and the second wave (from 14 September to 31 December 2020) in Italy.

In 2021 February/March, after the second wave, all HCWs (uninfected and previously infected) received the second dose of the vaccine because a specific recommendation to administer a single vaccine dose in previously infected subjects had not yet been issued.

After the second-dose administration of BNT162b2/ Pfizer, the mean level of RBD S-protein IgG was 30,559 AU/ $\mathrm{mL}(95 \%$ CI $145-80,000)$ in the previously infected HCWs, and $10,463 \mathrm{AU} / \mathrm{mL}(95 \%$ CI $165-62,241)(p<0.001)$ in uninfected HCWs. 
Table 1 Demographic characteristics and seroconversion level of 1510 care workers by antibodies against the SARS-CoV-2 status prior vaccine and to 45 days post-complete vaccination

\begin{tabular}{|c|c|c|c|}
\hline \multicolumn{4}{|l|}{ Prior SARS-CoV-2 infection } \\
\hline & $\begin{array}{l}\text { Yes } \\
(n=284)^{\mathrm{a}}\end{array}$ & $\begin{array}{l}\text { No } \\
(n=1226)^{\mathrm{b}}\end{array}$ & $p$ value \\
\hline Age, mean (range), years & $45(21-71)$ & $44(20-75)$ & ns \\
\hline \multicolumn{4}{|l|}{ Sex } \\
\hline Female, No. (\%) & $201(71)$ & $884(72)$ & ns \\
\hline Male, No. (\%) & $83(29)$ & $342(28)$ & ns \\
\hline \multicolumn{4}{|l|}{ SARS-CoV-2 IgG level } \\
\hline $\begin{array}{l}\text { N-Protein } \operatorname{IgG}>1.4 \text { signal to } \\
\text { cutoff ratio, No. }(\%)\end{array}$ & $284(100)$ & 0 & $<0.001$ \\
\hline $\begin{array}{l}\text { RBD S-Protein IgG antibody } \\
\text { mean, AU/mL }(95 \% \text { CI })\end{array}$ & $30,559(145-80,000)$ & $10,463(165-62,241)$ & $<0.001$ \\
\hline
\end{tabular}

In 64/101 (63.3\%) RT-PCR-positive symptomatic HCWs during the first wave (from 21 February to 11 June 2020), the mean level of post-vaccinal RBD S-protein IgG was $34,590 \mathrm{AU} / \mathrm{mL}$ (95\% CI 9922-80,000). In comparison, the $37 / 101$ (36.6\%) symptomatic subjects with infection during the second wave (from 14 September to 31 December 2020) had a mean level of $14,104 \mathrm{AU} / \mathrm{mL}$ (95\% CI 145-50,255) $(p<0.001)$.

The N-Protein IgG antibodies were detected in all 1510 HCWs enrolled in the study: 284 were positive (previous SARS-CoV-2 infection) and 1226 (uninfected) were negative (see Table 1). Only in 168 out of 284 was it possible to evaluate the levels of the N-Protein IgG 3 times in 2020 (April, June and November) while in 116, it was not possible. Data show that $64 \mathrm{HCWs}$ were persistently seropositive for SARS-CoV-2N-Protein IgG in all three repeated antibodies test and they had mean RBD S-protein IgG level of 37,320 AU/mL (95\% CI 6107-80,000). In contrast, 104 subjects were positive only in the first or second test (previously N-Protein IgG positive) who maintained a postvaccinal RBD S-protein IgG mean level of $29,096 \mathrm{AU} / \mathrm{mL}$ $(95 \%$ CI 3566-80,000) $(p<0.001)$; these subjects had also a significantly higher mean level of RBD S-protein IgG than the uninfected HCWs $(29,096 \mathrm{AU} / \mathrm{mL}$ vs $10,463 \mathrm{AU} / \mathrm{mL}$; $p<0.001$ ).

A few studies have shown that vaccinees who were previously infected with SARS-CoV-2 had a significantly higher antibody response and the booster response was more effective when the vaccine was administered more than 3 months after the infection [2].

Our study suggests that two doses of BNT162b2 vaccine may provide a very high level of RBD S-protein IgG antibody in subjects previously infected with SARS-CoV-2 and perhaps a single dose of the vaccine could be sufficient.
In positive RT-PCR subjects, the post-vaccination antibody level was higher if the infection was acquired during the first wave, 8-11 months before testing, compared to those with more recent infection ( $<6$ months). In addition, after vaccination previously infected individuals - who had lost SARS-CoV-2N-Protein IgG-showed a significantly higher mean level of RBD S-protein IgG than the uninfected subjects. This is probably a consequence of the memory $\mathrm{B}$ cell and $\mathrm{T}$ cell response to natural viral infection [3].

In conclusion, our study confirms that naturally infected individuals with SARS-CoV-2 have a very significant higher level of RBD S-Protein IgG after the second-dose administration of BNT162b2/Pfizer vaccine. The timing of one single-dose administration could be after $>11$ months from prior SARS-CoV-2 infection when their natural immunological response is stabilized because they produced antibodies, memory $\mathrm{B}$ and $\mathrm{T}$ cells that should be protective for 10 months against re-exposure $[4,5]$. Perhaps a third booster dose of the vaccine is unnecessary in previously infected subjects. Further studies will be needed to define a quantitative protection threshold, the rate of decline of antiviral antibodies and the timing of single vaccine dose administration in prior SARS-CoV-2-infected subjects.

Acknowledgements We wish to thank all Health Care Workers at the Poliambulanza Foundation Hospital of Brescia (Italy) who participated in this study, and Alessandro Triboldi (General Director, Chair Officer Executive), Maria Lorena Bazzani (Health Care Department) and Giovanna Ghitti (Department of Occupational Medicine) for their support.

Author contributions RS conceived and designed the study. RG collected data and performed the analysis. RS and RG wrote the first draft. All the authors provided substantial scientific input to the manuscript. All the authors revised and agreed upon the final version of the manuscript. 


\section{Declarations}

Conflict of interest The authors declare that there are no conflicts of interest and no external funding was received.

Ethics committee approval Ethical approval was obtained from Provincial Ethic Committee (NP4478).

\section{References}

1. L'Huillier AG, Meyer B, Andrey DO, et al. Antibody persistence in the first 6 months following SARS-CoV-2 infection among hospital workers: a prospective longitudinal study. Clin Microbiol Infect. 2021;27:784. https://doi.org/10.1016/j.cmi.2021.01.005.
2. Anichini G, Terrosi C, Gandolfo C, et al. SARS-CoV-2 antibody response in persons with past natural infection. $\mathrm{N}$ Engl J Med. 2021;385:90-2. https://doi.org/10.1056/NEJMc2103825.

3. Turner JS, Kim W, Kalaidina E, et al. SARS-CoV-2 infection induced long-lived bone marrow plasma cells in humans. Nature. 2021. https://doi.org/10.1038/s41586-021-03647-4.

4. Jung JH, Rha M-S, Sa M, et al. SARS-CoV-2-specific T cell memory is sustained in COVID-19 convalescent patients for 10 months with successful development of stem cell-like memory T cell. Nat Commun. 2021;12:4043. https://doi.org/10.1038/ s41467-021-24377-1.

5. Gaebler C, Wang Z, Lorenzi JCC, et al. Evolution of antibody immunity to SARS-CoV-2. Nature. 2021;591:639-44. https://doi. org/10.1038/s41586-021-03207-w. 